COMMUNICATIONS IN

ANALYSIS AND GEOMETRY

Volume 13, Number 5, 929-938, 2005

\title{
On the weak limits of smooth maps for the Dirichlet energy between manifolds
}

\author{
Fengbo Hang
}

\begin{abstract}
We identify all the weak sequential limits of smooth maps in $W^{1,2}(M, N)$. In particular, this implies a necessary and sufficient topological condition for smooth maps to be weakly sequentially dense in $W^{1,2}(M, N)$.
\end{abstract}

\section{Introduction.}

Assume $M$ and $N$ are smooth compact Riemannian manifolds without boundary and they are embedded into $\mathbb{R}^{l}$ and $\mathbb{R}^{\bar{l}}$ respectively. The following spaces are of interest in the calculus of variations:

$$
\begin{aligned}
W^{1,2}(M, N)= & \left\{u \in W^{1,2}\left(M, \mathbb{R}^{\bar{l}}\right): u(x) \in N \text { a.e. } x \in M\right\}, \\
H_{W}^{1,2}(M, N)= & \left\{u \in W^{1,2}(M, N): \text { there exists a sequence } u_{i} \in C^{\infty}(M, N)\right. \\
& \text { such that } \left.u_{i} \rightarrow u \text { in } W^{1,2}(M, N)\right\} .
\end{aligned}
$$

For a brief history and detailed references on the study of analytical and topological issues related to these spaces, one may refer to $[2,3,7]$. In particular, it follows from Theorem 7.1 of [3] that a necessary condition for $H_{W}^{1,2}(M, N)=W^{1,2}(M, N)$ is that $M$ satisfies the 1-extension property with respect to $N$ (see Section 2.2 of [3] for a definition). It was conjectured in Section 7 of [3] that the 1-extension property is also sufficient for $H_{W}^{1,2}(M, N)=$ $W^{1,2}(M, N)$. In $[1,7]$, it was shown that $H_{W}^{1,2}(M, N)=W^{1,2}(M, N)$ when $\pi_{1}(M)=0$ or $\pi_{1}(N)=0$. Note that if $\pi_{1}(M)=0$ or $\pi_{1}(N)=0$, then $M$ satisfies the 1-extension property with respect to $N$. In Section 8 of [4], it was proved that the above conjecture is true under the additional assumption that $N$ satisfies the 2 -vanishing condition. The main aim of the present article is to confirm the conjecture in its full generality. More precisely, we have 
Theorem 1.1. Let $M^{n}$ and $N$ be smooth compact Riemannian manifolds without boundary $(n \geq 3)$. Take a Lipschitz triangulation $h: K \rightarrow M$, then

$$
\begin{aligned}
& H_{W}^{1,2}(M, N) \\
& \quad=\left\{u \in W^{1,2}(M, N): u_{\#, 2}(h) \text { has a continuous extension to } M \text { w.r.t. } N\right\} \\
& \quad=\left\{u \in W^{1,2}(M, N): u \text { may be connected to some smooth maps }\right\} .
\end{aligned}
$$

In addition, if $\alpha \in[M, N]$ satisfies $\left.\alpha \circ h\right|_{\left|K^{1}\right|}=u_{\#, 2}(h)$, then we may find a sequence of smooth maps $u_{i} \in C^{\infty}(M, N)$ such that $u_{i} \rightarrow u$ in $W^{1,2}(M, N),\left[u_{i}\right]=\alpha$ and $d u_{i} \rightarrow d u$ a.e..

Here, $u_{\#, 2}(h)$ is the 1-homotopy class defined by White [8] (see also Section 4 of [3]) and $[M, N]$ means all homotopy classes of maps from $M$ to $N$. It follows from Theorem 1.1 that

Corollary 1.2. Let $M^{n}$ and $N$ be smooth compact Riemannian manifolds without boundary and $n \geq 3$. Then smooth maps are weakly sequentially dense in $W^{1,2}(M, N)$ if and only if $M$ satisfies the 1-extension property with respect to $N$.

For $p \in[3, n-1]$ being a natural number, it remains a challenging open problem to find out whether the weak sequential density of smooth maps in $W^{1, p}(M, N)$ is equivalent to the condition that $M$ satisfies the $p-1$ extension property with respect to $N$. This was verified to be true under further topological assumptions on $N$ (see Section 8 of [4]). However, even for $W^{1,3}\left(S^{4}, S^{2}\right)$, it is still not known whether smooth maps are weakly sequentially dense. Some very interesting recent work on this space can be found in [5].

The paper is written as follows. In Section 2, we will present some technical lemmas. In Section 3, we will prove the above Theorem and Corollary.

\section{Some preparations.}

The following local result, which was proved by Pakzad and Riviere in [7], plays an important role in our discussion.

Theorem 2.1 ([7]). Let $N$ be a smooth compact Riemannian manifold. Assume $n \geq 3, B_{1}=B_{1}^{n}, f \in W^{1,2}\left(\partial B_{1}, N\right) \cap C\left(\partial B_{1}, N\right), f \sim$ const, $u \in$ $W^{1,2}\left(B_{1}, N\right),\left.u\right|_{\partial B_{1}}=f$, then there exists a sequence $u_{i} \in W^{1,2}\left(B_{1}, N\right) \cap$ $C\left(\bar{B}_{1}, N\right)$ such that $\left.u_{i}\right|_{\partial B_{1}}=f, u_{i} \rightarrow u$ in $W^{1,2}\left(B_{1}, N\right)$ and $d u_{i} \rightarrow d u$ a.e.. 
In addition, if $v \in W^{1,2}\left(B_{2} \backslash B_{1}, N\right) \cap C\left(\bar{B}_{2} \backslash B_{1}, N\right)$ satisfies $\left.v\right|_{\partial B_{1}}=f$ and $\left.v\right|_{\partial B_{2}} \equiv$ const, then we may estimate

$$
\int_{B_{1}}\left|d u_{i}\right|^{2} d \mathcal{H}^{n} \leq c(n, N)\left(\int_{B_{1}}|d u|^{2} d \mathcal{H}^{n}+\int_{B_{2} \backslash B_{1}}|d v|^{2} d \mathcal{H}^{n}\right) .
$$

For convenience, we will use those notations and concepts in Sections 2, 3 and 4 of [3]. The following Lemma is a rough version of Luckhaus's Lemma [6]. For readers' convenience, we sketch a proof of this simpler version using results from Section 3 of [3].

Lemma 2.2. Assume $M^{n}$ and $N$ are smooth compact Riemannian manifolds without boundary. Let $e>0,0<\delta<1, A>0$, then there exists an $\varepsilon=\varepsilon(e, \delta, A, M, N)>0$ such that for any $u, v \in W^{1,2}(M, N)$ with $|d u|_{L^{2}(M)},|d v|_{L^{2}(M)} \leq A$ and $|u-v|_{L^{2}(M)} \leq \varepsilon$, we may find a $w \in W^{1,2}(M \times(0, \delta), N)$ such that, in the trace sense $w(x, 0)=u(x)$, $w(x, \delta)=v(x)$ a.e. $x \in M$ and

$$
|d w|_{L^{2}(M \times(0, \delta))} \leq c(M) \sqrt{\delta}\left(|d u|_{L^{2}(M)}+|d v|_{L^{2}(M)}+e\right) .
$$

Proof. Let $\varepsilon_{M}>0$ be a small positive number such that

$$
V_{2 \varepsilon_{M}}(M)=\left\{x \in \mathbb{R}^{l}: d(x, M)<2 \varepsilon_{M}\right\}
$$

is a tubular neighborhood of $M$. Let $\pi_{M}: V_{2 \varepsilon_{M}}(M) \rightarrow M$ be the nearest point projection. Similarly, we have $\varepsilon_{N}, V_{2 \varepsilon_{N}}(N)$ and $\pi_{N}$ for $N$. Choose a Lipschitz cubeulation $h: K \rightarrow M$. We may assume each cell in $K$ is a cube of unit size. For $\xi \in B_{\varepsilon_{M}}^{l}, x \in|K|$, let $h_{\xi}(x)=\pi_{M}(h(x)+\xi)$. Assume $\varepsilon_{M}$ is small enough such that all $h_{\xi}$ 's are bi-Lipschitz maps. Set $m=\left[\frac{1}{\delta}\right]+1$, using $[0,1]=\cup_{i=1}^{m}\left[\frac{i-1}{m}, \frac{i}{m}\right]$, we may divide each $k$-cube in $K$ into $m^{k}$ small cubes. In particular, we get a subdivision of $K$, called $K_{m}$. It follows from Section 3 of [3] that for a.e. $\xi \in B_{\varepsilon_{M}}^{l}, u \circ h_{\xi}, v \circ h_{\xi} \in \mathcal{W}^{1,2}\left(K_{m}, N\right)$. Applying the estimates in Section 3 of [3] to each unit size $k$-cube in $\left|K_{m}^{k}\right|$, we get

$$
\begin{aligned}
& \int_{B_{\varepsilon_{M}}^{l}} d \mathcal{H}^{l}(\xi) \int_{\left|K_{m}^{k}\right|}\left|d\left(\left.u \circ h_{\xi}\right|_{\left|K_{m}^{k}\right|}\right)\right|^{2} d \mathcal{H}^{k} \leq c(M) \delta^{k-n}|d u|_{L^{2}(M)}^{2}, \\
& \int_{B_{\varepsilon_{M}}^{l}} d \mathcal{H}^{l}(\xi) \int_{\left|K_{m}^{k}\right|}\left|d\left(\left.v \circ h_{\xi}\right|_{\left|K_{m}^{k}\right|}\right)\right|^{2} d \mathcal{H}^{k} \leq c(M) \delta^{k-n}|d v|_{L^{2}(M)}^{2},
\end{aligned}
$$


and

$$
\begin{aligned}
& \left(\int_{B_{\varepsilon_{M}}^{l}}\left|u \circ h_{\xi}-v \circ h_{\xi}\right|_{L^{\infty}\left(\left|K_{m}^{1}\right|\right)}^{2} d \mathcal{H}^{l}(\xi)\right)^{\frac{1}{2}} \\
& \quad \leq c(\delta, M)\left(|d(u-v)|_{L^{2}(M)}^{\frac{3}{4}}|u-v|_{L^{2}(M)}^{\frac{1}{4}}+|u-v|_{L^{2}(M)}\right) \\
& \quad \leq c(\delta, A, M) \varepsilon^{\frac{1}{4}}
\end{aligned}
$$

By the mean value inequality, we may find a $\xi \in B_{\varepsilon_{M}}^{l}$ such that $u \circ h_{\xi}, v \circ h_{\xi} \in$ $\mathcal{W}^{1,2}\left(K_{m}, N\right)$,

$$
\left|u \circ h_{\xi}-v \circ h_{\xi}\right|_{L^{\infty}\left(\left|K_{m}^{1}\right|\right)} \leq c(\delta, A, M) \varepsilon^{\frac{1}{4}}<\varepsilon_{N} \quad \text { when } \varepsilon \text { is small enough, }
$$

and

$$
\begin{aligned}
& \int_{\left|K_{m}^{k}\right|}\left[\left|d\left(\left.u \circ h_{\xi}\right|_{\left|K_{m}^{k}\right|} \mid\right)\right|^{2}+\left|d\left(v \circ h_{\xi}\left|K_{m}^{k}\right|\right)\right|^{2}\right] d \mathcal{H}^{k} \\
& \quad \leq c(M) \delta^{k-n}\left(|d u|_{L^{2}(M)}^{2}+|d v|_{L^{2}(M)}^{2}\right)
\end{aligned}
$$

for $1 \leq k \leq n$. Fix a $\eta \in C^{\infty}(\mathbb{R}, \mathbb{R})$ such that $0 \leq \eta \leq 1,\left.\eta\right|_{\left(-\infty, \frac{1}{3}\right)}=1$ and $\left.\eta\right|_{\left(\frac{2}{3}, \infty\right)}=0$. Letting $f=u \circ h_{\xi}, g=v \circ h_{\xi}$, we will define $\phi:|K| \times[0, \delta] \rightarrow N$ inductively. First, set $\phi(x, 0)=f(x)$ and $\phi(x, \delta)=g(x)$ for $x \in|K|$. For $\Delta \in K_{m}^{1} \backslash K_{m}^{0}$, on $\Delta \times[0, \delta]$, we let

$$
\phi(x, t)=\pi_{N}\left(\eta\left(\frac{t}{\delta}\right) f(x)+\left(1-\eta\left(\frac{t}{\delta}\right)\right) g(x)\right) \quad x \in \Delta, 0 \leq t \leq \delta .
$$

For $\Delta \in K_{m}^{2} \backslash K_{m}^{1}$, let $y_{\Delta}$ be the center of $\Delta$, and define $\phi$ on $\Delta \times[0, \delta]$ as the homogeneous degree zero extension of $\left.\phi\right|_{\partial(\Delta \times[0, \delta])}$ with respect to $\left(y_{\Delta}, \frac{\delta}{2}\right)$. Next, we handle each 3 -cube, 4 -cube, $\cdots, n$-cube in a similar way. Calculations show that

$$
\begin{aligned}
& \int_{|K| \times[0, \delta]}|d \phi|^{2} d \mathcal{H}^{n+1} \\
& \leq c(n) \sum_{k=1}^{n} \delta^{n+1-k} \int_{\left|K_{m}^{k}\right|}\left[\left|d\left(\left.u \circ h_{\xi}\right|_{\left|K_{m}^{k}\right|} \mid\right)\right|^{2}+\left|d\left(\left.v \circ h_{\xi}\right|_{\left|K_{m}^{k}\right|}\right)\right|^{2}\right] d \mathcal{H}^{k} \\
& \quad+c(\delta, A, M) \varepsilon^{\frac{1}{2}} \\
& \quad \leq c(M) \delta\left(|d u|_{L^{2}(M)}^{2}+|d v|_{L^{2}(M)}^{2}+e^{2}\right)
\end{aligned}
$$


when $\varepsilon$ is small enough. Finally, $w: M \times[0, \delta] \rightarrow N$, defined by $w(x, t)=$ $\phi\left(h_{\xi}^{-1}(x), t\right)$, is the needed map.

Lemma 2.3. Assume $N$ is a smooth compact Riemannian manifold, $n \geq 2$, $B_{1}=B_{1}^{n}, u, v \in W^{1,2}\left(B_{1}, N\right)$ such that $\left.u\right|_{\partial B_{1}}=\left.v\right|_{\partial B_{1}}$. Define $w: B_{1} \times$ $(0,1) \rightarrow N$ by

$$
w(x, t)=\left\{\begin{array}{cc}
u(x), & x \in B_{1} \backslash B_{t} ; \\
u\left(\frac{t^{2}}{|x|} \frac{x}{|x|}\right), & x \in B_{t} \backslash B_{t^{2}} ; \\
v\left(\frac{x}{t^{2}}\right), & x \in B_{t^{2}} ;
\end{array}\right.
$$

then $w \in W^{1,2}\left(B_{1} \times(0,1), N\right)$ and

$$
|d w|_{L^{2}\left(B_{1} \times(0,1)\right)} \leq c(n)\left(|d u|_{L^{2}\left(B_{1}\right)}+|d v|_{L^{2}\left(B_{1}\right)}\right) .
$$

Proof. Note that

$$
|d w(x, t)| \leq\left\{\begin{array}{cc}
|d u(x)|, & t<|x| \\
c(n)\left|d u\left(\frac{t^{2}}{|x|} \frac{x}{|x|}\right)\right| \frac{t^{2}}{|x|^{2}}, & t^{2}<|x|<t \\
c(n)\left|d v\left(\frac{x}{t^{2}}\right)\right| \frac{1}{t^{2}}, & |x|<t^{2} .
\end{array}\right.
$$

Hence

$$
\begin{aligned}
& \int_{\substack{0<t<1 \\
t^{2}<|x|<t}}|d w(x, t)|^{2} d \mathcal{H}^{n+1}(x, t) \\
& \leq c(n) \int_{0}^{1} d t \int_{t^{2}}^{t} d r \int_{\partial B_{r}}\left|d u\left(\frac{t^{2}}{r^{2}} x\right)\right|^{2} \frac{t^{4}}{r^{4}} d \mathcal{H}^{n-1}(x) \\
& \quad=c(n) \int_{0}^{1} d t \int_{t}^{1} d s \int_{\partial B_{s}} \frac{t^{2(n-2)}}{s^{2(n-2)}}|d u(y)|^{2} d \mathcal{H}^{n-1}(y) \\
& \leq c(n)|d u|_{L^{2}\left(B_{1}\right)}^{2}
\end{aligned}
$$

and

$$
\begin{aligned}
& \int_{\substack{0<t<1 \\
|x|<t^{2}}}|d w(x, t)|^{2} d \mathcal{H}^{n+1}(x, t) \\
& \quad \leq c(n) \int_{0}^{1} d t \int_{B_{t^{2}}}\left|d v\left(\frac{x}{t^{2}}\right)\right|^{2} \frac{1}{t^{4}} d \mathcal{H}^{n}(x) \\
& \quad \leq c(n)|d v|_{L^{2}\left(B_{1}\right)}^{2} .
\end{aligned}
$$

The lemma follows. 


\section{Identifying weak limits of smooth maps.}

In this section, we shall prove Theorem 1.1 and Corollary 1.2.

Proof of Theorem 1.1. Let $h: K \rightarrow M$ be a Lipschitz cubeulation. We may assume each cell in $K$ is a cube of unit size. Let $\varepsilon_{M}>0$ be a small number such that

$$
V_{2 \varepsilon_{M}}(M)=\left\{x \in \mathbb{R}^{l}: d(x, N)<2 \varepsilon_{M}\right\}
$$

is a tubular neighborhood of $M$. Denote $\pi_{M}: V_{2 \varepsilon_{M}}(M) \rightarrow M$ as the nearest point projection. For $\xi \in B_{\varepsilon_{M}}^{l}$, we let $h_{\xi}(x)=\pi_{M}(h(x)+\xi)$ for $x \in|K|$, the polytope of $K$. We may assume $\varepsilon_{M}$ is small enough such that all $h_{\xi}$ are bi-Lipschitz maps. Replacing $h$ by $h_{\xi}$ when necessary, we may assume $f=u \circ h \in \mathcal{W}^{1,2}(K, N)$. Then we may find a $g \in C(|K|, N) \cap \mathcal{W}^{1,2}(K, N)$ such that $\left[g \circ h^{-1}\right]=\alpha$ and $\left.g\right|_{\left|K^{1}\right|}=\left.f\right|_{\left|K^{1}\right|}$ (see the proofs of Theorem 5.5 and Theorem 6.1 in [4]). For each cell $\Delta \in K$, let $y_{\Delta}$ be the center of $\Delta$. For $x \in \Delta$, let $|x|_{\Delta}$ be the Minkowski norm with respect to $y_{\Delta}$, that is

$$
|x|_{\Delta}=\inf \left\{t>0: y_{\Delta}+\frac{x-y_{\Delta}}{t} \in \Delta\right\} \text {. }
$$

Step 1: For every $\Delta \in K^{2} \backslash K^{1}$, we may find a sequence $\phi_{i} \in C(\Delta, N) \cap$ $W^{1,2}(\Delta, N)$ such that $\left.\phi_{i}\right|_{\partial \Delta}=\left.g\right|_{\partial \Delta},\left.\phi_{i} \rightarrow f\right|_{\Delta}$ in $W^{1,2}(\Delta, N)$ and $d \phi_{i} \rightarrow$ $d\left(\left.f\right|_{\Delta}\right)$ a.e. (see Lemma 4.4 in [3]). For $x \in \Delta$, let

$$
f_{i}(x)=\left\{\begin{array}{cc}
\phi_{i}(x), & |x|_{\Delta} \geq \frac{1}{2^{i}} ; \\
\phi_{i}\left(y_{\Delta}+\frac{1}{2^{2 i}|x|_{\Delta}} \frac{x-y_{\Delta}}{|x|_{\Delta}}\right), & \frac{1}{2^{2 i}} \leq|x|_{\Delta} \leq \frac{1}{2^{i}} ; \\
g\left(y_{\Delta}+2^{2 i}\left(x-y_{\Delta}\right)\right), & |x|_{\Delta} \leq \frac{1}{2^{2 i}} .
\end{array}\right.
$$

It is clear that $\left.f_{i} \rightarrow f\right|_{\Delta}$ in $W^{1,2}(\Delta, N), d f_{i} \rightarrow d\left(\left.f\right|_{\Delta}\right)$ a.e. on $\Delta$,

$$
\left|d f_{i}\right|_{L^{2}(\Delta)} \leq c \cdot\left(\left|d \phi_{i}\right|_{L^{2}(\Delta)}+\left|d\left(\left.g\right|_{\Delta}\right)\right|_{L^{2}(\Delta)}\right) \leq c(f, g)
$$

and $f_{i} \in C\left(\left|K^{2}\right|, N\right)$. In addition, if we define $h_{2, i}: \Delta \times[0,1] \rightarrow N$ by

$$
h_{2, i}(x, t)=\left\{\begin{array}{cc}
\phi_{i}(x), & |x|_{\Delta} \geq \frac{1}{2^{i}}+\frac{2^{i}-1}{2^{i}} t ; \\
\phi_{i}\left(y_{\Delta}+\frac{\left(\frac{1}{2^{i}}+\frac{2^{i}-1}{2^{i}} t\right)^{2}}{|x|_{\Delta}} \frac{x-y_{\Delta}}{|x|_{\Delta}}\right), & \left(\frac{1}{2^{i}}+\frac{2^{i}-1}{2^{i}} t\right)^{2} \leq|x|_{\Delta} \\
& \leq \frac{1}{2^{i}}+\frac{2^{i}-1}{2^{i}} t ; \\
g\left(y_{\Delta}+\frac{x-y_{\Delta}}{\left(\frac{1}{2^{i}}+\frac{2^{i}-1}{2^{i}} t\right)^{2}}\right), & |x|_{\Delta} \leq\left(\frac{1}{2^{i}}+\frac{2^{i}-1}{2^{i}} t\right)^{2} .
\end{array}\right.
$$


Then by Lemma 2.3 , we know $h_{2, i} \in W^{1,2}(\Delta \times[0,1], N)$,

$$
\left|d h_{2, i}\right|_{L^{2}(\Delta \times[0,1])} \leq c \cdot\left(\left|d \phi_{i}\right|_{L^{2}(\Delta)}+\left|d\left(\left.g\right|_{\Delta}\right)\right|_{L^{2}(\Delta)}\right) \leq c(f, g)
$$

and $h_{2, i} \in C\left(\left|K^{2}\right| \times[0,1], N\right)$.

Step 2: Assume for some $2 \leq k \leq n-1$, we have a sequence $f_{i} \in$ $C\left(\left|K^{k}\right|, N\right) \cap \mathcal{W}^{1,2}\left(K^{k}, N\right)$ and $h_{k, i} \in C\left(\left|K^{k}\right| \times[0,1], N\right)$ such that for each $\Delta \in K^{k},\left.f_{i} \rightarrow f\right|_{\Delta}$ in $W^{1,2}(\Delta, N), h_{k, i} \in W^{1,2}(\Delta \times[0,1], N)$,

$$
\left|d\left(\left.f_{i}\right|_{\Delta}\right)\right|_{L^{2}(\Delta)} \leq c(f, g), \quad\left|d h_{k, i}\right|_{L^{2}(\Delta \times[0,1])} \leq c(f, g)
$$

and $h_{k, i}(x, 0)=f_{i}(x), h_{k, i}(x, 1)=g(x)$ for $x \in\left|K^{k}\right|$. Since for every $\Delta \in K^{k+1} \backslash K^{k},\left.f_{i} \rightarrow f\right|_{\partial \Delta}$ in $W^{1,2}(\partial \Delta, N)$, for fixed $j$ by Lemma 2.2 we may find a $n_{j} \geq j$ such that for each $\Delta \in K^{k+1} \backslash K^{k}$, there exists a $w_{j} \in W^{1,2}\left(\partial \Delta \times\left[0,2^{-j}\right], N\right)$ with $w_{j}(x, 0)=f(x), w_{j}\left(x, \frac{1}{2^{j}}\right)=f_{n_{j}}(x)$ and

$$
\left|d w_{j}\right|_{L^{2}\left(\partial \Delta \times\left(0, \frac{1}{2^{j}}\right)\right)} \leq \frac{c(n)}{2^{\frac{j}{2}}}\left(\left|d\left(\left.f\right|_{\partial \Delta}\right)\right|_{L^{2}(\partial \Delta)}+\left|d f_{n_{j}}\right|_{L^{2}(\partial \Delta)}+1\right) \leq \frac{c(f, g)}{2^{\frac{j}{2}}} .
$$

Without loss of generality, we may replace $f_{i}$ by $f_{n_{i}}$ and $h_{k, i}$ by $h_{k, n_{i}}$. Fix a $\Delta \in K^{k+1} \backslash K^{k}$. For $x \in \Delta$, let

$$
\psi_{i}(x)=\left\{\begin{array}{cc}
f\left(y_{\Delta}+\frac{2^{i}\left(x-y_{\Delta}\right)}{2^{i}-1}\right), & |x|_{\Delta} \leq \frac{2^{i}-1}{2^{i}} \\
w_{i}\left(y_{\Delta}+\frac{x-y_{\Delta}}{|x|_{\Delta}},|x|_{\Delta}-\frac{2^{i}-1}{2^{i}}\right), & \frac{2^{i}-1}{2^{i}} \leq|x|_{\Delta} \leq 1 .
\end{array}\right.
$$

Then $\left.\psi_{i}\right|_{\left|K^{k}\right|}=f_{i}$ and $\left.\psi_{i} \rightarrow f\right|_{\Delta}$ in $W^{1,2}(\Delta, N)$ as $i \rightarrow \infty$ for each $\Delta \in K^{k+1} \backslash K^{k}$. By Theorem 2.1 and (3.1) (use $h_{k, i}$ and $g$ for the needed " $v$ " in Theorem 2.1, one may refer to Lemma 9.8 of [4]), for every $\Delta \in K^{k+1} \backslash K^{k}$, we may find $\phi_{i} \in C(\Delta, N) \cap W^{1,2}(\Delta, N)$ such that $\left.\phi_{i}\right|_{\partial \Delta}=\left.f_{i}\right|_{\partial \Delta}$, $\left|\phi_{i}-\psi_{i}\right|_{L^{2}(\Delta)}<\frac{1}{2^{i}},\left|d \phi_{i}\right|_{L^{2}(\Delta)} \leq c(f, g)$ and

$$
\int_{M} \frac{\left|d \phi_{i}-d \psi_{i}\right|}{1+\left|d \phi_{i}-d \psi_{i}\right|} d \mathcal{H}^{k+1} \leq \frac{1}{2^{i}}
$$

After passing to subsequence, we may assume $d \phi_{i} \rightarrow d\left(\left.f\right|_{\Delta}\right)$ a.e. on $\Delta$. Fix 
a $\Delta \in K^{k+1} \backslash K^{k}$, for any $x \in \Delta$, define

$$
\begin{gathered}
g_{k+1, i}(x)=\left\{\begin{array}{cc}
h_{k, i}\left(y_{\Delta}+\frac{x-y_{\Delta}}{|x|_{\Delta}}, 1+2\left(\frac{1}{2}-|x|_{\Delta}\right)\right), & \frac{1}{2} \leq|x|_{\Delta} \leq 1 ; \\
g\left(y_{\Delta}+2\left(x-y_{\Delta}\right)\right), & |x|_{\Delta} \leq \frac{1}{2},
\end{array}\right. \\
f_{i}(x)=\left\{\begin{array}{cc}
\phi_{i}(x), & |x|_{\Delta} \geq \frac{1}{2^{i}} ; \\
\phi_{i}\left(y_{\Delta}+\frac{1}{2^{2 i}|x|_{\Delta}} \frac{x-y_{\Delta}}{|x|_{\Delta}}\right), & \frac{1}{2^{2 i}} \leq|x|_{\Delta} \leq \frac{1}{2^{i}} ; \\
g_{k+1, i}\left(y_{\Delta}+2^{2 i}\left(x-y_{\Delta}\right)\right), & |x|_{\Delta} \leq \frac{1}{2^{2 i}},
\end{array}\right. \\
\widetilde{h}_{k+1, i}(x, t)=\left\{\begin{array}{cc}
\phi_{i}(x), & |x|_{\Delta} \geq \frac{1}{2^{i}}+\frac{2^{i}-1}{2^{i}} t ; \\
\phi_{i}\left(y_{\Delta}+\frac{\left(\frac{1}{2^{i}}+\frac{2^{i}-1}{2^{i}} t\right)^{2}}{|x|_{\Delta}} \frac{x-y_{\Delta}}{|x|_{\Delta}}\right), & \left(\frac{1}{2^{i}}+\frac{2^{i}-1}{2^{i}} t\right)^{2} \leq|x|_{\Delta}
\end{array}\right. \\
\widetilde{\widetilde{h}}_{k+1, i}(x, t)=\left\{\begin{array}{cc}
g_{k+1, i}\left(y_{\Delta}+\frac{1}{2^{i}}+\frac{2^{i}-1}{2^{i}} t ;\right. \\
\left.h_{k, i}\left(y_{\Delta}+\frac{x-y_{\Delta}}{\left.2^{i}+\frac{2^{i}-1}{2^{i}} t\right)^{2}}\right), 1+2\left(\frac{1+t}{2}-|x|_{\Delta}\right)\right), & \frac{1+t}{2} \leq|x|_{\Delta} \leq\left(\frac{1}{2^{i}}+\frac{2^{i}-1}{2^{i}} t\right)^{2}, \\
g\left(y_{\Delta}+\frac{2}{1+t}\left(x-y_{\Delta}\right)\right), & |x|_{\Delta} \leq \frac{1+t}{2},
\end{array}\right.
\end{gathered}
$$

and

$$
h_{k+1, i}(x, t)=\left\{\begin{array}{cc}
\widetilde{h}_{k+1, i}(x, 2 t), & 0 \leq t \leq \frac{1}{2} ; \\
\widetilde{\widetilde{h}}_{k+1, i}(x, 2 t-1), & \frac{1}{2} \leq t \leq 1 .
\end{array}\right.
$$

Simple calculations show that for any $\Delta \in K^{k+1} \backslash K^{k},\left.f_{i} \rightarrow f\right|_{\Delta}$ in $W^{1,2}(\Delta, N), d f_{i} \rightarrow d\left(\left.f\right|_{\Delta}\right)$ a.e. on $\Delta, h_{k+1, i} \in W^{1,2}(\Delta \times[0,1], N)$,

$$
\left|d f_{i}\right|_{L^{2}(\Delta)} \leq c(f, g), \quad\left|d h_{k+1, i}\right|_{L^{2}(\Delta \times[0,1])} \leq c(f, g)
$$

and $h_{k+1, i}(x, 0)=f_{i}(x), h_{k+1, i}(x, 1)=g(x)$ for $x \in\left|K^{k+1}\right|$. Hence, we finish when we reach $f_{i} \in C(|K|, N) \cap \mathcal{W}^{1,2}(K, N)$ and $h_{n, i} \in$ $C(|K| \times[0,1], N)$. Let $v_{i}=f_{i} \circ h^{-1}$. Then it is clear that $v_{i} \in C(M, N) \cap$ $W^{1,2}(M, N),\left[v_{i}\right]=\alpha,\left|v_{i}-u\right|_{L^{2}(M)} \rightarrow 0,\left|d v_{i}\right|_{L^{2}(M)} \leq c(u, g)$ and $d v_{i} \rightarrow d u$ a.e. on $M$. Hence, we may find $u_{i} \in C^{\infty}(M, N)$ such that $\left|u_{i}-u\right|_{L^{2}(M)} \rightarrow 0$, $\left|d u_{i}\right|_{L^{2}(M)} \leq c(u, g),\left[u_{i}\right]=\alpha$ and $d u_{i} \rightarrow d u$ a.e. on $M$. In particular, this shows

$$
\begin{gathered}
H_{W}^{1,2}(M, N) \supset\left\{u \in W^{1,2}(M, N): u_{\#, 2}(h)\right. \text { has a continuous extension to } \\
M \text { w.r.t. } N\} .
\end{gathered}
$$

The other direction of inclusion was proved in Section 7 of [3]. To see

$$
\begin{gathered}
H_{W}^{1,2}(M, N)=\left\{u \in W^{1,2}(M, N): u\right. \text { may be connected to some smooth } \\
\text { maps }\},
\end{gathered}
$$


we only need to use the above proved equality and proposition 5.2 of [3], which shows

$$
\begin{gathered}
\left\{u \in W^{1,2}(M, N): u_{\#, 2}(h) \text { has a continuous extension to } M \text { w.r.t. } N\right\} \\
=\left\{u \in W^{1,2}(M, N): u \text { may be connected to some smooth maps }\right\}
\end{gathered}
$$

We remark that many constructions above are motivated from Sections 5 and 6 of [4].

Proof of Corollary 1.2. This follows from Theorem 1.1 and Corollary 5.4 of $[3]$.

\section{Acknowledgments.}

The research of the author is supported by National Science Foundation Grant DMS-0209504.

\section{References.}

[1] P. Hajlasz. Approximation of Sobolev mappings. Nonlinear Anal 22(12) (1994), 1579-1591.

[2] F. B. Hang and F. H. Lin. Topology of Sobolev mappings. Math Res Lett 8(3) (2001), 321-330.

[3] F. B. Hang and F. H. Lin. Topology of Sobolev mappings II. Acta Math 191(1) (2003), 55-107.

[4] F. B. Hang and F. H. Lin. Topology of Sobolev mappings III. Comm Pure Appl Math 56 (10) (2003), 1383-1415.

[5] R. Hardt and T. Riviere. Connecting topological Hopf singularities. Annali Sc Norm Sup Pisa, 2(2) (2003), 287-344.

[6] S. Luckhaus. Partial Holder continuity for minima of certain energies among maps into a Riemannian manifold. Indiana Univ Math $J \mathbf{3 7}$ (1988), 349-367.

[7] M. R. Pakzad and T. Riviere. Weak density of smooth maps for the Dirichlet energy between manifolds. Geom Func Anal 13(1) (2003), 223257. 
[8] B. White. Homotopy classes in Sobolev spaces and the existence of energy minimizing maps. Acta Math 160(1-2) (1988), 1-17.

Department of Mathematics

Princeton University

Fine Hall, Washington Road

PRINCETON, NJ 08544

AND

School of Mathematics

Institute For AdVANCED StUdy

1 Einstein Drive, Princeton, NJ 08540

E-mail address: fhang@math.princeton.edu

Received December 5, 2003. 